\title{
Alternative measures of uncertainty in quantum metrology: Contradictions and limits
}

\author{
Alfredo Luis* and Alfonso Rodil \\ Departamento de Óptica, Facultad de Ciencias Físicas, Universidad Complutense, 28040 Madrid, Spain
}

(Received 25 November 2012; published 7 March 2013)

\begin{abstract}
We examine a family of intrinsic performance measures in terms of probability distributions that generalize Hellinger distance and Fisher information. They are applied to quantum metrology to assess the uncertainty in the detection of minute changes of physical quantities. We show that different measures lead to contradictory conclusions, including the possibility of arbitrarily small uncertainty for fixed resources. These intrinsic performances are compared with the averaged error in the corresponding estimation problem after single-shot measurements.
\end{abstract}

DOI: 10.1103/PhysRevA.87.034101

PACS number(s): 03.65.Ta, 42.50.St, 42.50.Lc, 89.70.Cf

\section{INTRODUCTION}

Quantum fluctuations and uncertainty are key issues in quantum physics because of the fundamental statistical nature of the theory. They also enter on practical matters such as quantum metrology, where it is of fundamental importance to determine whether quantum fluctuations impose an ultimate lower limit to the uncertainty in the detection of minute changes of physical quantities. The evidence supporting the universality of a lower bound known as the Heisenberg limit [1] is not as solid as would be desirable as revealed by recent examples [2]. In some very recent approaches more conclusive results were obtained by averaging uncertainty over finite intervals for the monitored variable representing our prior knowledge about it [3].

Historically, the statistical inference about uncertainty is addressed by variance-based methods, mainly because they properly fit Gaussian statistics. Nevertheless, this may be not satisfactory enough in other situations, and alternative approaches may be of interest [4-11]. Previous works have already shown that different assessments of fluctuations may lead to contradictory and counterintuitive conclusions. For example, states with diverging variance may have arbitrarily small entropy for the very same observable [9]. This ambiguity extends to the uncertainty relation between complementary observables when using Renyi-Tsallis entropic measures, since the very same state can be either of maximum or of minimum joint uncertainty, depending on the measure used [10]. Moreover, for some entropic uncertainty measures there is no lower bound to the joint uncertainty of complementary observables [11] (see also Ref. [12]).

After these precedents we think that it is worth investigating the application of alternative measures of uncertainty to the question of fundamental resolution limits in quantum metrology caused by quantum uncertainty. To this end we address an intrinsic evaluation of the detection performance in terms of the closeness between the probability distributions associated with two close enough values of the monitored variable [13]. We use Renyi-Tsallis generalizations of the Hellinger distance and Fisher information. This is compared with the averaged error in the corresponding estimation problem.

\footnotetext{
*alluis@ fis.ucm.es; URL: http://www.ucm.es/info/gioq
}

\section{GENERALIZED DISTANCES}

In general terms, a signal variable $\epsilon$ is monitored by the transformation $P \rightarrow P_{\epsilon}$ it induces in some observed quantity $P$. Within a quantum context we consider that $P=P(x)$ is the probability distribution of a given observable $X$, which will be assumed dimensionless, continuous, and unbounded, as a coordinate of a particle or a field quadrature, for example. Nevertheless, $P$ may equally well represent any other quantity in classical or quantum physics, such as the intensity of a light beam in classical optics, for example.

We focus on the very usual case where the signal-induced transformation $P \rightarrow P_{\epsilon}$ is just a shift $P(x) \rightarrow P(x-\epsilon)$. The significance of $\epsilon$ can be assessed by the closeness between $P(x)$ and $P(x-\epsilon)[14]$ :

$$
D_{q}=\frac{1}{2} \int_{-\infty}^{\infty} d x\left|P^{q}(x-\epsilon)-P^{q}(x)\right|^{1 / q},
$$

where $q$ is any positive real number. These are Renyi-Tsallis generalizations of the Hellinger distance, which is the case $q=1 / 2$ [8]. We may regard $P^{q}$ as a kind of nonlinear record of $P$. This can be illustrated by the example of $P$ as light intensity where $P^{q}$ for integer $q>1$ may represent the nonlinear optical response of a nonlinear medium. On the other hand, $q=1 / 2$ is the case of homodyne detection, where the detector current is proportional to the amplitude of the signal electric field.

For weak signals $\epsilon \ll 1$ (the case of major interest in precision metrology) we have, to first order in $\epsilon$,

$$
D_{q} \simeq \frac{q^{1 / q}}{2}|\epsilon|^{1 / q} F_{q}, \quad F_{q}=\int_{-\infty}^{\infty} d x P(x)\left|\frac{d}{d x} \ln P(x)\right|^{1 / q} .
$$

Therefore, $F_{q}$ is a family of generalizations of the Fisher information as $1 / q$ moments of the score, where the Fisher information is retrieved for $q=1 / 2$ [15].

This provides an estimation of detection sensitivity as the minimum signal $\epsilon_{\min }$ required to exceed some threshold for $D_{q}$. For simplicity, we assume that such a threshold does not depend on the probe state, so that the sensitivity is solely determined by $F_{q}$ in the form

$$
\epsilon_{\min }=\frac{1}{F_{q}^{q}}
$$

We have evaluated the generalized $F_{q}$ at $\epsilon=0$. Nevertheless, the conclusions so obtained extend to other $\epsilon$ values 


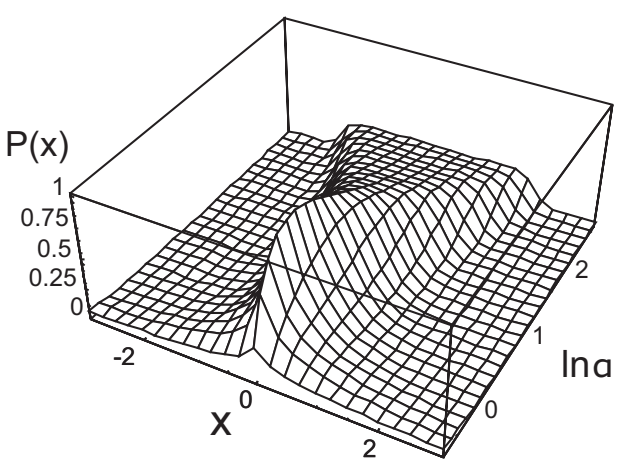

FIG. 1. Three-dimensional plot of $P(x)$ in Eq. (4) as a function of $x$ and $\ln \alpha$ with $\gamma$ given by Eq. (5) with $\langle E\rangle=1$. Throughout this work plotted quantities are dimensionless.

because the signal-induced transformation is just a shift that does not affect the form of the probability distribution. This differs from other transformations, such as optical phase shifts, that change the form of the measured distribution for most probe states and practical observables, leading to Fisher information depending on the value of the signal. This is essentially the reason for averaging uncertainties over prescribed prior intervals for the monitored variable carried out in Ref. [3].

In the general case, $D_{q}^{q}$ is not a proper distance for $q \neq 1 / 2$, since the triangle inequality may fail. Nevertheless, regarding metrological applications we are just interested in the $\epsilon$ dependence around $\epsilon=0$, where, after Eq. (2), $D_{q}^{q} \propto|\epsilon| F_{q}^{q}$ behaves as a proper distance.

\section{PROBE STATE}

Let us consider probe states following an exponential power distribution (also referred to as generalized normal distribution, or generalized error distribution) illustrated in Fig. 1, i.e.,

$$
P(x)=\frac{\alpha 2^{1 / \alpha}}{2 \gamma \Gamma(1 / \alpha)} \exp \left(-2|x / \gamma|^{\alpha}\right),
$$

where $\alpha$ and $\gamma$ are real non-negative parameters. For $\alpha=1$ this is the bound state of delta potentials $V(x) \propto-\delta(x)$, while for $\alpha=2$ these are Gaussians including the fundamental state of harmonic oscillators, $V(x) \propto x^{2}$. On the other hand, for $\alpha \rightarrow \infty, P(x)$ tends to be a square distribution, which for a free particle may be implemented with suitably arranged shutters acting on a momentum eigenstate [16]. We assume that the signal-dependent transformation and the measurement are fast enough so we do not have to consider the free evolution of the probe during the process.

The parameter $\gamma$ may be conveniently expressed in terms of the mean value of the energy $\langle E\rangle$, according to the usual practice in quantum metrology of relating resolution with the energy resources employed [1-3]. For simplicity, let us assume that the probe is a free particle $E=p^{2}$, where $p$ represents the dimensionless variable canonically conjugate to $x$. From now on we consider the energetically optimum case where the wave function is real, $\psi(x)=\sqrt{P(x)}$, so that $\langle p\rangle=0$. Then, the computation of $\langle E\rangle=(\Delta p)^{2}=\left\langle p^{2}\right\rangle$ readily provides a

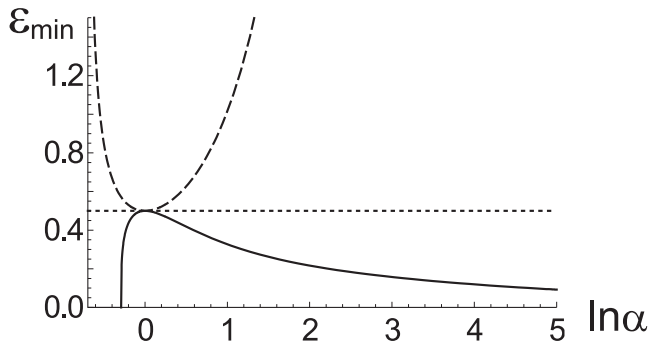

FIG. 2. $\epsilon_{\min }$ for fixed $\langle E\rangle=1$ as a function of $\ln \alpha$ for $q=1 / 4$ (solid line), $q=1 / 2$ (dotted line), and $q=2$ (dashed line).

relation between $\gamma$ and the mean energy $\langle E\rangle$ valid for $\alpha>1 / 2$

$$
\gamma=\frac{\alpha 2^{1 / \alpha}}{2 \sqrt{\langle E\rangle}} \sqrt{\frac{\Gamma(2-1 / \alpha)}{\Gamma(1 / \alpha)} .}
$$

The case $\langle p\rangle=0$ is energetically optimum because the sensitivity to $x$ shifts will depend on $\Delta x$ and $\Delta p$, but not on $\langle x\rangle$ or $\langle p\rangle$. Thus, the condition $\langle p\rangle=0$ avoids expending energy resources on dynamical states not related to the efficiency of the detection.

\section{ALTERNATIVE MEASURES}

Next, the intrinsic performance assessment in Eq. (3) is compared with the estimation uncertainty after the measurement of $X$. In all cases we follow the standard practice in quantum metrology of analyzing detection performances for fixed energy resources [1-3]. We focus on single-shot measurement since it is energetically optimum to concentrate all resources in a single measurement [1].

\section{A. Generalization of Fisher information}

By direct computation of $F_{q}$ after Eqs. (2) and (4) we get for $\alpha>\max (1-q, 1 / 2)$

$$
F_{q}=\frac{\Gamma\left(\frac{\alpha+q-1}{\alpha q}\right)}{\Gamma(1 / \alpha)}\left(\frac{\alpha 2^{1 / \alpha}}{\gamma}\right)^{1 / q} .
$$

After Eqs. (3), (5), and (6) we get

$$
\epsilon_{\min }=\frac{\Gamma^{q}(1 / \alpha)}{\alpha 2^{1 / \alpha} \Gamma^{q}\left(\frac{\alpha+q-1}{q \alpha}\right)} \gamma .
$$

In Fig. 2 we have plotted $\epsilon_{\min }$ as a function of $\alpha$ for several values of $q$. We can appreciate strong differences arising for different values of $q$ and $\alpha$. For $q>1 / 2$ we have $\epsilon_{\min } \rightarrow \infty$ for

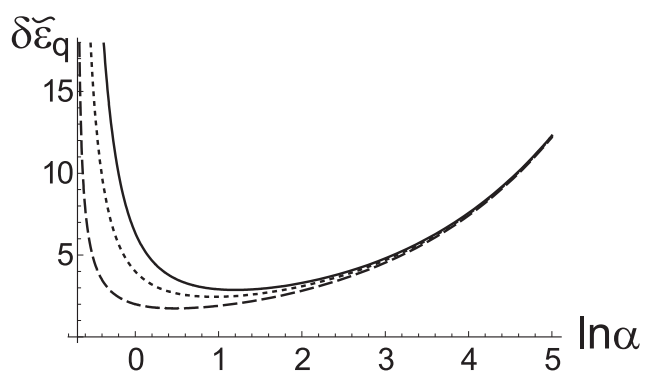

FIG. 3. $\delta \tilde{\epsilon}_{q}$ in Eq. (8) as function of $\alpha$ for fixed $\langle E\rangle=1$ and $q=1 / 4$ (solid line), $q=1 / 2$ (dotted line), and $q=2$ (dashed line). 


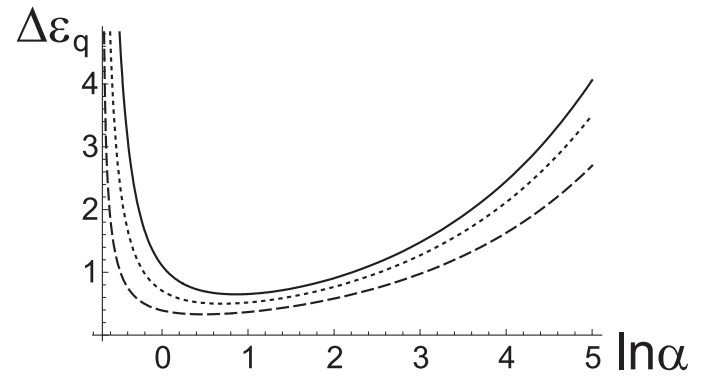

FIG. 4. $\Delta \epsilon_{q}$ in Eq. (9) as function of $\alpha$ for fixed $\langle E\rangle=1$ and $q=1 / 4$ (solid line), $q=1 / 2$ (dotted line), and $q=2$ (dashed line).

both $\alpha \rightarrow 1 / 2$ and $\alpha \rightarrow \infty$, with $\epsilon_{\min }$ being the minimum for $\alpha=1$. On the other hand, for $q<1 / 2$ we have the exact opposite behavior with $\epsilon_{\min } \rightarrow 0$ for both $\alpha \rightarrow 1-q$ and $\alpha \rightarrow \infty$, providing $\alpha=1$ as the maximum for $\epsilon_{\min }$. Finally, for $q=1 / 2$ there is no dependence on $\alpha$.

\section{B. Width of posterior distribution}

From a Bayesian perspective, after any outcome $x$ we can infer a conditional probability distribution for the estimate $\tilde{\epsilon}$ of $\epsilon$ as $P(\tilde{\epsilon} \mid x)=P(x-\tilde{\epsilon})$. A suitable measure of the estimation uncertainty is given by the width of $P(\tilde{\epsilon} \mid x)$ as a function of $\tilde{\epsilon}$. Following the spirit of the preceding sections we may consider Renyi-Tsallis measures of uncertainty $\delta \tilde{\epsilon}_{q}$ (or generalized Fisher lengths) as [12,17]

$$
\delta \tilde{\epsilon}_{q}=\left[\int d x P^{q}(\tilde{\epsilon} \mid x)\right]^{\frac{1}{1-q}}=\frac{1}{q^{\frac{1}{\alpha(1-q)}}} \frac{2 \Gamma(1 / \alpha)}{\alpha 2^{1 / \alpha}} \gamma,
$$

which are independent of $x$. In Fig. 3 we have represented $\delta \tilde{\epsilon}_{q}$ showing that it is quite similar for all values of $q$ examined, and also very similar to the case $q>1 / 2$ of $\epsilon_{\min }$.

\section{Mean estimation error}

After a single observation the outcome $x$ is a suitable unbiased estimator of $\epsilon$ since its average coincides with the true value $\int d x \times P(x \mid \epsilon)=\epsilon$, where $P(x \mid \epsilon)=P(x-\epsilon)$ is the probability of $x$ conditioned to $\epsilon$. In the same spirit of the above generalization we may consider the mean estimation error

$\Delta \epsilon_{q}=\left[\int d x P(x \mid \epsilon)|x-\epsilon|^{1 / q}\right]^{q}=\frac{1}{2^{1 / \alpha}} \frac{\Gamma^{q}\left(\frac{1+q}{\alpha q}\right)}{\Gamma^{q}(1 / \alpha)} \gamma$,

which is independent of $\epsilon$. In Fig. 4 we have represented $\Delta \epsilon_{q}$ showing that it is quite similar to $\delta \tilde{\epsilon}_{q}$ for all values of $q$ examined.

\section{DISCUSSION AND CONCLUSIONS}

We have examined the assessment of uncertainty provided by a family of generalizations of the Hellinger distance and the Fisher information. After considering probes in exponential power distributions we have obtained two relevant conclusions:

(i) Different measures lead to contradictory conclusions. This is illustrated by the cases $q=1 / 4$ and $q=2$ in Fig. 2 where $\alpha=1$ provides the maximum sensitivity for $q>1 / 2$ and the minimum sensitivity for $q<1 / 2$.

(ii) For $q<1 / 2$ we get increasingly high sensitivity for probes with properly chosen values of $\alpha$ for fixed and finite resources.

We have computed also similarly generalized averaged errors in the corresponding single-shot estimation problem, showing that they do not reproduce the two above features. Thus we wonder whether there is any suitable counterpart of the Cramer-Rao lower bound involving these generalized performance measures.

We think that this approach may provide useful insights for the understanding of uncertainty, uncertainty relations, and their implications in quantum metrology. The vanishing of $\epsilon_{\min }$ for finite energy resources that arise for $q<1 / 2$ seems to defy basic ideas in quantum metrology about ultimate resolution limits. It would be of interest to determine whether this result is related to the lack of lower bound to the joint uncertainty of incompatible observables that arises for related Renyi-Tsallis uncertainty measures [11]. On the other hand, in previous works we have found that the relation between quantum limits and uncertainty relations is not trivial since probe states leading to minimum metrological uncertainty may be far from being minimum uncertainty states [18].

The result (ii) also recalls the increasing sensitivity of Fabry-Perot arrangements for increasing mirror reflectivity [19]. This might be regarded as a kind of instrumental factor provided by the probe state that can be exploited for some values of $q$. Moreover, the ambiguity reported in conclusion (i) might be solved if physical reasons might impose that the performance measures used should be adapted to the probe state considered in each case.

\section{ACKNOWLEDGMENTS}

A.L. acknowledges support from Projects No. FIS201235583 of the Spanish Ministerio de Economía y Competitividad, and No. QUITEMAD S2009-ESP-1594 of the Comunidad de Madrid.
[1] V. Giovannetti, S. Lloyd, and L. Maccone, Phys. Rev. Lett. 96, 010401 (2006); M. Zwierz, C. A. Perez-Delgado, and P. Kok, ibid. 105, 180402 (2010); V. Giovannetti, S. Lloyd, and L. Maccone, Science 306, 1330 (2004)

[2] A. Rivas and A. Luis, New J. Phys. 14, 093052 (2012).

[3] M. Tsang, Phys. Rev. Lett. 108, 230401 (2012); V. Giovannetti and L. Maccone, ibid. 108, 210404 (2012); Y. Gao and H. Lee, J. Phys. A 45, 415306 (2012); V. Giovannetti, S. Lloyd, and
L. Maccone, Phys. Rev. Lett. 108, 260405 (2012); M. J. W. Hall, D. W. Berry, M. Zwierz, and H. M. Wiseman, Phys. Rev. A 85, 041802(R) (2012); M. J. W. Hall and H. M. Wiseman, New J. Phys. 14, 033040 (2012); R. Nair, arXiv:1204.3761v1.

[4] J. Hilgevoord, Am. J. Phys. 70, 983 (2002); G. N. Lawrence, Laser Focus World 30, 109 (1994); J. Řeháček and Z. Hradil, J. Mod. Opt. 51, 979 (2004). 
[5] A. Luis, Phys. Rev. A 64, 012103 (2001); 67, 032108 (2003).

[6] J.-M. Lévy-Leblond, Ann. Phys. (NY) 101, 319 (1976); E. Breitenberger, Found. Phys. 15, 353 (1985); J. B. M. Uffink, Phys. Lett. A 108, 59 (1985); J.-M. Lévy-Leblond, ibid. 111, 353 (1985); S. M. Barnett and D. T. Pegg, J. Mod. Opt. 36, 7 (1989); Z. Hradil, Phys. Rev. A 46, R2217 (1992); Quantum Opt. 4, 93 (1992); T. Opatrný, J. Phys. A 27, 7201 (1994); V. Peřinová, A. Lukš, and J. Peřina, Phase in Optics (World Scientific, Singapore, 1998).

[7] I. I. Hirschman, Am. J. Math. 79, 152 (1957); I. BialynickiBirula and J. Mycielski, Commun. Math. Phys. 44, 129 (1975); D. Deutsch, Phys. Rev. Lett. 50, 631 (1983); M. H. Partovi, ibid. 50, 1883 (1983); K. Kraus, Phys. Rev. D 35, 3070 (1987); H. Maassen and J. B. M. Uffink, Phys. Rev. Lett. 60, 1103 (1988); J. Sanchez, Phys. Lett. A 173, 233 (1993); Č. Brukner and A. Zeilinger, Phys. Rev. Lett. 83, 3354 (1999); Phys. Rev. A 63, 022113 (2001); V. Majerník and E. Majerníková, Rep. Math. Phys. 47, 381 (2001); M. J. W. Hall, Phys. Rev. A 64, 052103 (2001); S. Massar and P. Spindel, Phys. Rev. Lett. 100, 190401 (2008); S. Wehner and A. Winter, New J. Phys. 12, 025009 (2010); I. Bialynicki-Birula and L. Rudnicki arXiv:1001.4668v1; I. Urizar-Lanz and G. Tóth, Phys. Rev. A 81, 052108 (2010); A. D. C. Nascimento, R. J. Cintra, and A. C. Frery, IEEE Trans. Geos. Remot. Sens. 48, 373 (2010); P. Sánchez-Moreno, A. R. Plastino, and J. S. Dehesa, J. Phys. A 44, 065301 (2011); A. E. Rastegin, ibid. 44, 095303 (2011).

[8] A. Renyi, in Proceedings of the 4th Berkeley Symposium on Mathematics and Statistical Probability, Vol. 1 (University of California Press, Berkeley, 1961), pp. 547-561; C. Tsallis, J. Stat. Phys. 52, 479 (1988); U. Larsen, J. Phys. A 23, 1041 (1990); E. M. F. Curado and C. Tsallis, ibid. 24, L69 (1991);
A. K. Rajagopal, Phys. Lett. A 205, 32 (1995); I. Csiszár, IEEE Trans. Inf. Theory 41, 26 (1995); M. Portesi and A. Plastino, Physica A 225, 412 (1996); I. Bialynicki-Birula, Phys. Rev. A 74, 052101 (2006); F. Chapeau-Blondeau, A. Delahaies, and D. Rousseau, Phys. Lett. A 375, 2211 (2011).

[9] P. Matía-Hernando and A. Luis, Phys. Rev. A 86, 052106 (2012). [10] A. Luis, Phys. Rev. A 84, 034101 (2011).

[11] M. Zakai, Inf. Control 3, 101 (1960); A. Luis, Opt. Lett. 31, 3644 (2006); Phys. Rev. A 75, 052115 (2007); S. Zozor, M. Portesi, and C. Vignat, Physica A 387, 4800 (2008).

[12] M. J. W. Hall, Phys. Rev. A 62, 012107 (2000).

[13] Z. Hradil, R. Myška, T. Opatrný, and J. Bajer, Phys. Rev. A 53, 3738 (1996); G. A. Durkin and J. P. Dowling, Phys. Rev. Lett. 99, 070801 (2007).

[14] N. Hadjisavvas, Lin. Alegbra Appl. 84, 281 (1986); Z.-H. Ma and J.-L. Chen, J. Phys. A 44, 195303 (2011).

[15] H. Cramér, Mathematical Methods of Statistics (Asia Publishing House, Bombay, 1962); T. M. Cover and J. A. Thomas, Elements of Information Theory (Wiley Interscience, New York, 1991); C. W. Helstrom, Phys. Lett. A 25, 101 (1967); IEEE Trans. Inf. Theory IT-14, 234 (1968); Quantum Detection and Estimation Theory (Academic, New York, 1976); S. L. Braunstein and C. M. Caves, Phys. Rev. Lett. 72, 3439 (1994).

[16] M. Moshinsky, Phys. Rev. 88, 625 (1952); E. Torrontegui, J. Muñoz, Y. Ban, and J. G. Muga, Phys. Rev. A 83, 043608 (2011).

[17] M. J. W. Hall, Phys. Rev. A 59, 2602 (1999).

[18] D. Maldonado-Mundo and A. Luis, Phys. Rev. A 80, 063811 (2009); A. Luis, Ann. Phys. (NY) 331, 1 (2013).

[19] A. Luis and L. L. Sánchez-Soto, J. Mod. Opt. 38, 971 (1991). 